\title{
Modeling of a solar receiver-reactor for sulfur-based thermochemical cycles for hydrogen generation
}

\author{
Adam Noglik, Martin Roeb*,† Christian Sattler and Robert Pitz-Paal \\ German Aerospace Center (DLR), Institute of Technical Thermodynamics, Solar Research, 51170 Köln, Germany
}

\section{SUMMARY}

Sulfur-based thermochemical cycles for hydrogen generation from water have one reaction step in common, which is the decomposition of sulfuric acid, which is one of the most energy-consuming steps. The present work deals with the development of a dynamic mathematical model of a solar reactor for this key step. One of the core parts of the model is a partial model of the reaction kinetics of the decomposition of sulfur trioxide, which is based on experiments investigating the kinetics of the used catalyst platinum coated on a ceramic solar absorber. Other partial models describe, e.g. the absorption of solar radiation, heat conduction in the absorber, convection between gas and the absorber walls and energy losses due to heat radiation.

A comprehensive validation of the reactor model is performed using measured data, which is gained in experiments with a prototype reactor. The operating behavior of the real reactor is compared with the results of the numerical simulation with the model. The validation is, in particular, performed by reproducing the influences of individual parameters on the chemical conversion and the reactor efficiency. The relative deviations between the experimental data and the simulation results are mostly within the range of measurement accuracy. In particular, the good agreement of calculated values of the derived parameters, $\mathrm{SO}_{3}$ conversion and reactor efficiency with those determined from the experiments qualifies the model for optimization purposes. Copyright (C) 2010 John Wiley \& Sons, Ltd.

\section{KEY WORDS}

solar chemistry; reactor modeling; sulfuric acid decomposition; solar energy; concentrated solar radiation; sulfur iodine cycle; hybrid sulfur cycle

Correspondence

*Martin Roeb, German Aerospace Center (DLR), Institute of Technical Thermodynamics, Solar Research, 51170 Köln, Germany.

†E-mail: martin.roeb@dlr.de

Contract/grant sponsor: European Commission; contract/grant number: 502704

Received 27 August 2009; Revised 9 March 2010; Accepted 10 March 2010

\section{INTRODUCTION}

Hydrogen is an environmentally attractive energy carrier with a long-term potential to replace fossil fuels in many applications. In particular this is true, if hydrogen is produced with reduced or even better completely without $\mathrm{CO}_{2}$ emissions. This can be realized by the use of renewable energy sources in the production process [1].

Only water and biomass are viable long-term candidate raw materials for regenerative hydrogen production. Thermochemical cycles and electrolysis have the greatest likelihood of successful massive hydrogen production from water. In the thermochemical processes, water is decomposed into hydrogen and oxygen via chemical reactions using intermediate substances, which are recycled. The use of solar thermal energy for covering the heat demand of these thermochemical processes allows to reach the high temperatures needed.

One important objective of the EU FP6 project HYTHEC was to improve the potential for hydrogen production using the Sulfur-Iodine cycle (SI) [2] or the Hybrid-Sulfur (HyS) cycle [3] driven by the solar energy. The most promising concept for delivering sufficient energy at a sufficiently high temperature to a massive, industrial scale SI or HyS production unit is a solar central receiver system (CRS), consisting of a large number of sun-tracking mirrors, the so-called 
heliostats and a receiver for the concentrated radiation situated on the top of a tower.

Process concepts for a solar operation of both cycles [4] suitable for the application in a CRS consider a receiver-reactor, which is simultaneously a receiver for absorbing the concentrated radiation from a heliostat field and a reactor for the decomposition of sulfuric acid. This concept offers less heat transferring steps and therewith a potential for higher efficiencies in comparison with other concepts using a heat transfer medium. The reactor was developed and qualified for tests in the solar furnace of DLR in Cologne [5]. The receiver-reactor presented in earlier studies [6-8] included both reaction steps of sulfuric acid decomposition, evaporation of sulfuric acid and $\mathrm{SO}_{3}$ decomposition, in one reaction compartment. One central finding was that this strategy imposes strong limitations on the developoment and optimization of both individual steps. The decision was taken to modifiy the receiver-reactor such that it is dedicated only to the solar $\mathrm{SO}_{3}$ decomposition.

The modified reactor has been extensively experimentally tested in the solar furnace. The aspects concerning the reactor development and the results of the experimental series have been published earlier in [9-12]. The objective of the present paper is to introduce a numerical model that has been developed for this version of the receiver-reactor and its validations.

\section{BACKGROUND}

Different concepts to bring in solar heat into a endothermal chemical process and in particular into the decomposition of sulfuric acid have been discussed in the past. Those concepts differ with respect to the coupling of the chemical process to the solar energy source. In some concepts, the radiation is directly absorbed by the reactants itself, e.g. a volumetric receiver-reactor has been proposed by Knoche [13]. Lüpfert performed first experiments in the solar furnace regarding the decomposition of waste sulfuric acid and found an enhancement of the decomposition of sufur trioxide by a photonic effect [14]. General Atomcis developed and tested a metallic tube reactor with a cavity design, where radiation is absorbed by the tubes and converted to heat, which is transferred to the reactants through the tube walls [15]. Owing to the material, its operating temperature was limited to about $900^{\circ} \mathrm{C}$ and therewith its efficiency.

Other concepts make use of inert heat transfer media that are heated in a solar receiver and transport the heat to the reactants itself - directly by mixing or via heat exchangers [16]. Alternative to that is the use of solar receivers in combination with heat-exchanger converters without mixing of heat transfer fluid and reactants. The heated fluid is transported to a thermochemical plant for hydrogen generation. For that purpose, additional heat exchanger and heat-exchanger converters are necessary (see, e.g. [17]). The advantage is the separation of heat collection and chemical conversion, which allows for an optimization of both steps individually and offers flexibility during intermittent operation. The efficiency is generally lower compared with direct absorbing receiver-reactors due to the losses in those heat exchangers and in pipes. A new variant of this concept was introduced by Kolb et al. [18] proposing sand instead of an inert gas, which enables the simultaneous use as an absorbing medium, as a heat transfer medium and as a storage medium. Challenges in this concept are the development of a sand receiver and the transfer of the heat from the solid particles to the thermochemical process.

Offering the potential of achieving the highest efficiencies, it was decided to investigate and put into practice and verify the potential of a representative concept of a direct absorbing receiver-reactor. A volumetric receiver-reactor was realized by our group for the direct solar decomposition of sulfuric acid.

The decomposition of sulfuric acid proceeds in several steps. First, the acid has to be vaporized followed by a decomposition of the gaseous $\mathrm{H}_{2} \mathrm{SO}_{4}$ into $\mathrm{H}_{2} \mathrm{O}$ and $\mathrm{SO}_{3}$, which is almost completed above $600^{\circ} \mathrm{C}$ :

$$
\begin{array}{r}
\mathrm{H}_{2} \mathrm{SO}_{4(\mathrm{~g})} \rightleftharpoons \mathrm{H}_{2} \mathrm{O}_{(\mathrm{g})}+\mathrm{SO}_{3(\mathrm{~g})} \\
\Delta H_{\mathrm{R}}^{0}=+97.5 \mathrm{~kJ} \mathrm{~mol}^{-1}
\end{array}
$$

Thereafter $\mathrm{SO}_{3}$ dissociates into $\mathrm{SO}_{2}$ and $\mathrm{O}_{2}$. This part reaction is almost completed above $1300^{\circ} \mathrm{C}$.

$$
\begin{aligned}
\mathrm{SO}_{3(\mathrm{~g})} \rightleftharpoons \mathrm{SO}_{2(\mathrm{~g})}+\frac{1}{2} \mathrm{O}_{2(\mathrm{~g})} \\
\Delta H_{\mathrm{R}}^{0}=+98.95 \mathrm{~kJ} \mathrm{~mol}^{-1}
\end{aligned}
$$

Solar concentrating technologies enable to provide the necessary heat at those temperature levels. The most recent version of the reactor (Figure 1) is specialized on the second step, the reduction of $\mathrm{SO}_{3}$. The reactors' main purpose was to investigate and optimize only the high-temperature step, the decomposition of

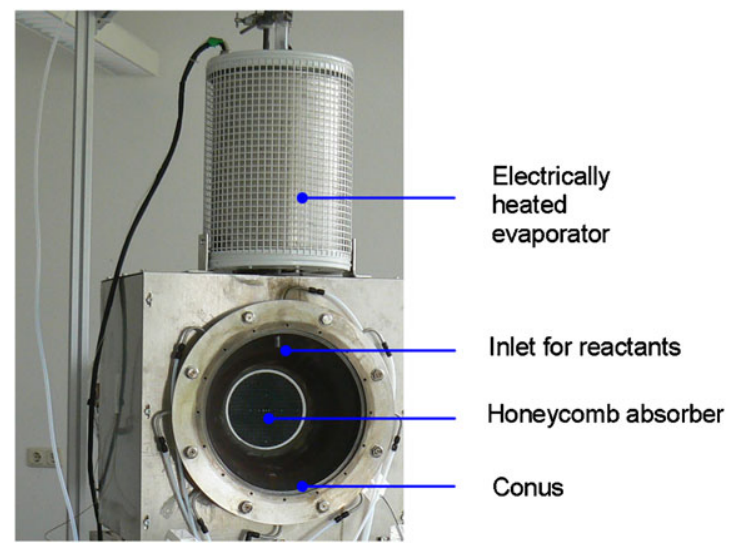

Figure 1. Front view of solar receiver-reactor [12]. 
$\mathrm{SO}_{3}$ by concentrated solar radiation. The upstream step of sulfuric acid evaporation is operated by an electrically heated reactor located on the top of the solar receiver-reactor for $\mathrm{SO}_{3}$ reduction. A gaseous mixture of $\mathrm{H}_{2} \mathrm{SO}_{4}, \mathrm{SO}_{3}, \mathrm{H}_{2} \mathrm{O}$ and $\mathrm{N}_{2}$ as carrier gas is fed into the solar part of the prototype reactor. The total mass flow through the reactor is in the range of $0.25-8 \mathrm{~g} \mathrm{~s}^{-1}$. The solar powered reaction, the $\mathrm{SO}_{3}$ reduction, is carried out inside a ceramic honeycomb absorber, whose front side is irradiated and heated by concentrated sunlight. The absorber has a diameter of $14.4 \mathrm{~cm}$ and a length of $15 \mathrm{~cm}$. The channels have a width of $2 \mathrm{~mm}$. The quartz window has a diameter of $30 \mathrm{~cm}$. A conical-shaped duct made of high-temperature-resistant steel encloses the reactor between the quartz pane and the absorber (Figure 2). The reaction takes place at temperatures between 800 and $1200^{\circ} \mathrm{C}$. In particular, near the lower boundary of this range the use of catalysts is essential.

As only a part of the operational points of the reaction, which are in particular interesting for a potential scale up, were accessible due to limitations in the experimental setup and experimental time, the development of a numerical model of the receiver-reactor became necessary. Modeling and simulation of the reactor enable the optimization of the reactor and of the operating parameters. It thereby facilitates the assessment of this technology in general.

\section{REACTOR MODELING}

The numerical reactor model consists of a set of sub models which represent the separate physical and chemical processes in the reactor. The basis was a

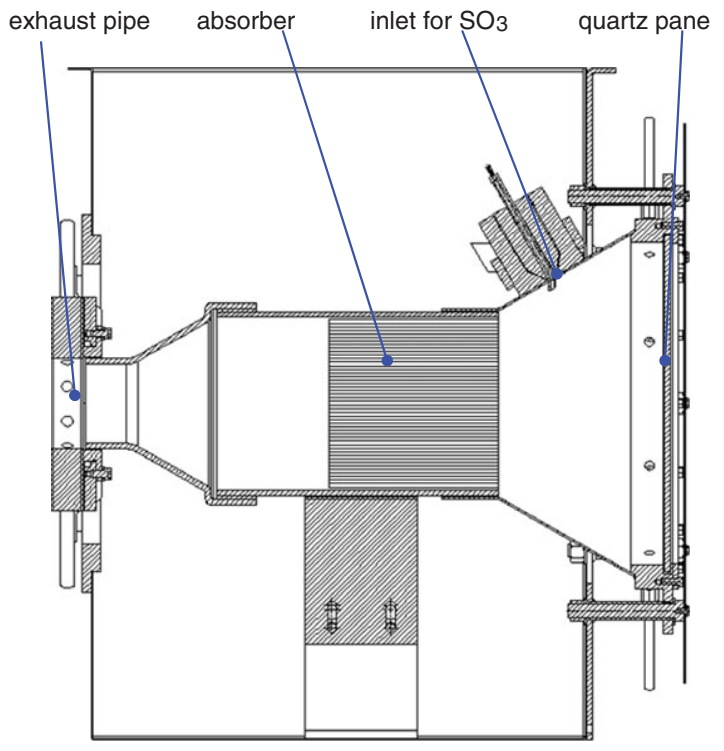

Figure 2. Scheme of solar receiver-reactor. model of a similar reactor dedicated to the generation of hydrogen from water by metal oxides $[19,20]$. The model was built in Dymola using partial models from the standard library Modelica 2.2 in combination with the Modelica_Fluid library and additionally adapted or newly developed models [21].

The structure of the overall model enables a specification of the following process values as input parameters:

- Solar irradiated power $P_{\mathrm{S}}$.

- Mass flow of sulfuric acid $\dot{m}_{\text {sulfuric acid }}$

- Mass fraction of sulfuric acid $w$.

- Temperature of the inlet flow $T_{\text {in }}$.

- Mass flow of the carrier gas at window $\dot{m}_{\mathrm{N}_{2}, I}$.

- Mass flow of the carrier gas into the vaporizer $\dot{m}_{\mathrm{N}_{2}, I I}$.

- Length of the absorber $l_{\text {Absorber }}$.

The main output parameters are the conversion of $\mathrm{SO}_{3}$ in the reactor, reactor efficiency, operating temperature, outlet temperature and residence time. Beyond these main output parameters all those parameters are calculated which are necessary for a verification and interpretation of the simulation results, e.g. concentration and mass flow of all reagents and products involved, temperature gradients of the absorber, etc.

\subsection{Absorber model}

3.1.1. Discretization. The cylindrical solar absorber is a ceramic honeycomb structure with more than two thousand channels through which the process gas flows and where the reaction takes place. Simulating each channel individually results in numerical difficulties. Therefore, the absorber is modeled as a homogeneous solid body. The porosity of the absorber is considered using effective material properties.

The absorber is discretized as shown in Figure 3. In the axial direction it is partitioned into 10 slices. Each slice is further partitioned into seven rings, where the center

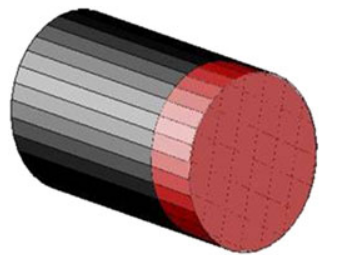

Absorber

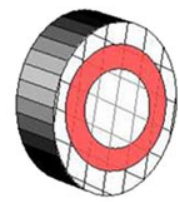

Slice of the Absorber (axial discretization)

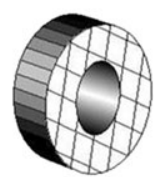

Ring of the Slice (radial discretization)

Figure 3. Scheme of the absorber discretization in the axial and radial direction [19]. 
rings are cylinders. Such a discretization results in 70 volume elements. The temperature in each of those elements is regarded as constant. One of the assumptions is the axis-symmetry of system results in a twodimensional model. The $10 \times 7$ discretization has been chosen in a study in which different grid sizes have been compared. It was not possible to use a higher number of elements due to numerical limitations of the used simulation system. Other combinations such as $11 \times 6$ or $8 \times 8$ were also possible but showed worse results. A lower number of elements resulted in a significant difference between the experimental data and the simulation results.

The elements have different sizes. The thickness of the discs increases from the first disc at the irradiated side to the back side of the absorber, see Figure 4. This is advantageous for the simulation since the temperature gradient in the axial direction decreases from the front to the end of the absorber. The radial thickness which is the difference between the outer radius and the inner radius of each ring is the same. The center elements have a diameter that is twice the radial thickness of the other elements.

3.1.2. Model of the volume elements. The volume elements for the gas and for the solid body are modeled separately, but linked by interfaces enabling transfer of energy. As the temperature in each volume element is constant over the volume (but still timedependent), material properties such as heat capacity and heat conductivity are constant as well.

Each volume element is connected to its neighbor elements. The energy balance for one solid volume element can be given as

$$
\begin{aligned}
0= & m \cdot c_{p} \cdot \frac{\partial T}{\partial t}+\dot{Q}_{\text {cond }, a, \text { radial }}+\dot{Q}_{\text {cond }, b, \text { radial }}+\dot{Q}_{\text {cond }, c, \text { axial }} \\
& +\dot{Q}_{\text {cond }, d, \text { axial }}+\dot{Q}_{\text {rad }, a, \text { radial }}+\dot{Q}_{\text {rad }, b, \text { radial }}+\dot{Q}_{\text {solar }} \\
& -\dot{Q}_{\text {convection }}
\end{aligned}
$$

where the term $m \cdot c_{p} \cdot \partial T / \partial t$ represents the heat storage in the element. $\dot{Q}_{\text {cond }}$ and $\dot{Q}_{\text {rad }}$ represent the conductive and radiative heat flows from or to adjacent cells in the radial and axial direction of the cylindrical

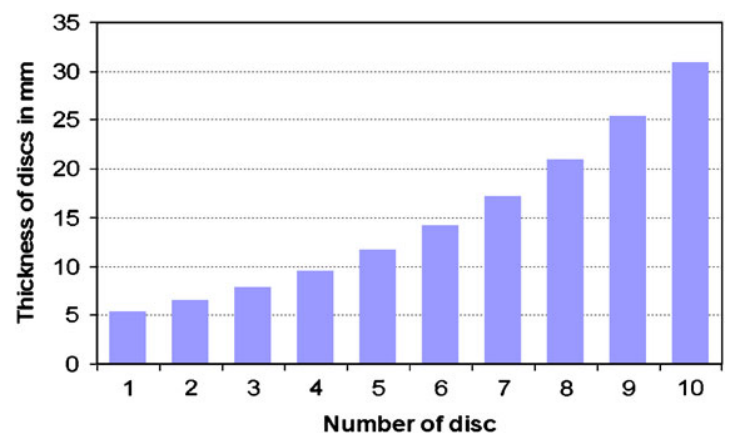

Figure 4. Increasing thickness of discs in the axial direction. absorber. E.g. $\dot{Q}_{\text {cond, } a \text {,radial }}$ is the heat conduction heat flow in the radial direction stemming from the neighbor element closer to the absorber front, $\dot{Q}_{\text {rad }, b \text {,radial }}$ is the radiation heat flow to the next element toward the absorber rim. $\dot{Q}_{\text {solar }}$ is the absorbed heat flow from the solar radiation and $\dot{Q}_{\text {convection }}$ is the heat flow transferred to the gas by forced convection. The heat balance of an inner solid volume element is shown in Figure 5. Subscript ' $a$ ' determines the neighbor element toward the front end, ' $b$ ' the neighbor element toward the rear face of the absorber.

For a gas volume element, the energy balance is as follows:

$$
0=\dot{m}_{\text {in }} \cdot h_{\text {in }}-\dot{m}_{\text {out }} \cdot h_{\text {out }}-\dot{Q}_{\text {reaction }}+\dot{Q}_{\text {convection }}
$$

where the terms $\dot{m}_{\text {in }} \cdot h_{\text {in }}$ and $\dot{m}_{\text {out }} \cdot h_{\text {out }}$ specify the enthalpy flows of the gas at the inlet and outlet of the volume element, $\dot{Q}_{\text {reaction }}$ is the heat flow consumed by the chemical reaction.

The solid volume elements at the rear face of the absorber do not have neighbour elements further to the back. Thus, there is no heat conduction in axial direction any more. Instead they lose energy due to thermal radiation (TR) to the reducer tube in the reactor and the ambience.

3.1.3. Model of the chemical reaction. The reduction of sulfur trioxide is performed inside the absorber, where the solar energy is transferred to the gas. In spite of the high temperatures of upto $1200^{\circ} \mathrm{C}$, the reaction rate is rather low when operating without a catalyst. Hence, the channels in the absorber are coated with platinum, which results in fast reaction kinetics. It is assumed that the gases do not react outside the absorber.

Furthermore, it is assumed that the dissociation of $\mathrm{H}_{2} \mathrm{SO}_{4}$ into $\mathrm{SO}_{3}$ and $\mathrm{H}_{2} \mathrm{O}$ occurs directly after inlet into the absorber as this reaction proceeds instantaneously.

A channel of the absorber can be considered as a plug-flow-reactor. In this ideal reactor type, the reaction velocity is dependant on the position in the

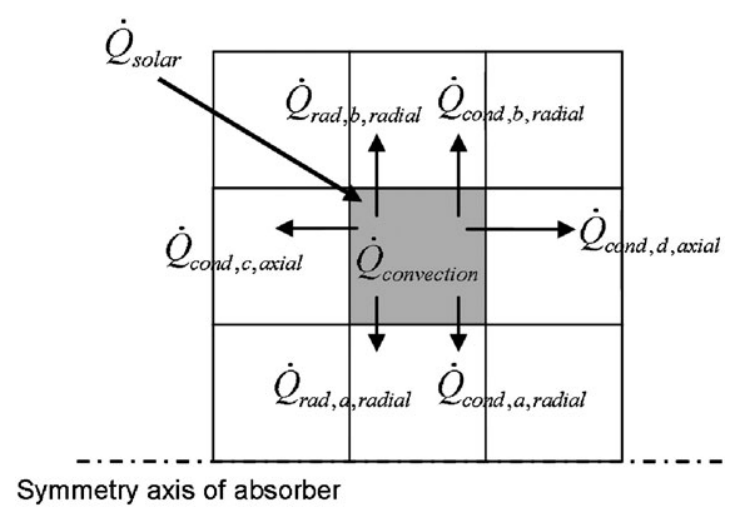

Figure 5. Sketch for the energy balance for one solid volume element with inlet and outlet heat flows. 
channel. A plug-flow-reactor can be modeled as a serial connection of continuously stirred tank reactors. The channel is partitioned into a sufficient number of stirred tank reactors with constant reaction velocities and concentrations in each reactor. Such a modeling approach fits the discretization of the absorber but due to the high-temperature gradients, the number of volume elements in the axial direction is not sufficient for an apropriate consideration of the reaction kinetics. The number of volume elements in the discretization cannot be further increased due to numerical problems.

To solve this problem, each volume element is divided into sub-cells only for the calculation of the reaction kinetics. The sufficient number of sub-cells per volume element turned out to be 20 . In every sub-cell of one volume element, the temperature is the same but the gas concentrations are changing from one cell to the next cell.

The reaction velocity $v_{[i]}$ in the sub-cell $i$ is calculated by the following equation:

$$
\begin{aligned}
v_{[i]}= & k \cdot\left(x_{\mathrm{SO}_{3}, \text { out }[i-1]} \cdot c_{\mathrm{gas}}\right)^{n} \mathrm{SO}_{3}-k_{\text {back }} \\
& \cdot\left(\left(x_{\mathrm{SO}_{2}, \text { out }[i-1]} \cdot c_{\mathrm{gas}}\right)^{n} \mathrm{SO}_{2} \cdot\left(x_{\mathrm{O}_{2}, \text { out }[i-1]} \cdot c_{\mathrm{gas}}\right)^{n} \mathrm{O}_{2}\right)
\end{aligned}
$$

where $k$ and $k_{\text {back }}$ are the reaction rate coefficients of the $\mathrm{SO}_{3}$ decomposition and the back reaction of the $\mathrm{SO}_{2}$-oxidation. $c_{\text {gas }}$ is the molar concentration in the volume element given in $\mathrm{mol} \mathrm{Nm}^{-3} \cdot x_{\mathrm{SO}_{3} \text {,out }[i-1]}$ is the molar fraction of $\mathrm{SO}_{3}$ at the outlet of the upstream sub-cell. $n_{\mathrm{SO}_{3}}$ is the reaction order of $\mathrm{SO}_{3}$ in the $\mathrm{SO}_{3}$ decomposition reaction. $n_{\mathrm{SO}_{2}}$ and $n_{\mathrm{O}_{2}}$ are the reaction orders of $\mathrm{SO}_{2}$ and $\mathrm{O}_{2}$ in the $\mathrm{SO}_{2}$-oxidation reaction. The missing reaction orders are zero.

The reaction rate coefficients are calculated with the Arrhenius equation:

$$
k=k_{\infty, 1} \cdot \mathrm{e}^{-E_{a, 1} / R \cdot T}, \quad k_{\text {back }}=k_{\infty, 2} \cdot \mathrm{e}^{-E_{a, 2} / R \cdot T}
$$

where $k_{\infty, 1}$ and $k_{\infty, 2}$ are the pre-exponential factors, $E_{a, 1}$ and $E_{a, 2}$ are the activation energies, $T$ is the temperature in the volume element and $R$ is the gas constant.

The kinetic parameters $n_{\mathrm{SO}_{3}}, n_{\mathrm{SO}_{2}}, n_{\mathrm{O}_{2}}, k_{\infty, 1}, E_{a, 1}$, $k_{\infty, 1}$ and $E_{a, 2}$ have been experimentally determined as described in [22] (Table I). A tube reactor was used for that purpose. The catalyst was platinum coated on an $\mathrm{SiC}$ honeycomb. By measuring the initial reaction rates for different temperatures and different reactant concentrations, it was possible to derive the parameter given in Table I for the $\mathrm{SO}_{3}$ reduction and for the $\mathrm{SO}_{2}$ oxidation (back reaction).

\subsection{Model of the solar radiation}

Concentrated solar radiation is the only power source for the reactor. The characteristics of the concentrated radiation not only determine the power input into the
Table I. Kinetic parameters used in the model.

\begin{tabular}{lccccccc}
\hline Para-meter & $n_{\mathrm{SO}_{3}}$ & $n_{\mathrm{SO}_{2}}$ & $n_{\mathrm{O}_{2}}$ & $k_{\infty, 1}$ & $E_{a, 1}$ & $k_{\infty, 2}$ & $E_{a, 2}$ \\
\hline Unit & & & & $\mathrm{s}^{-1}$ & $\mathrm{~kJ} \mathrm{~mol}^{-1}$ & $\mathrm{~s}^{-1}$ & $\mathrm{~kJ} \mathrm{~mol}^{-1}$ \\
Value & 0.86 & 1.5 & 1.5 & 2154 & 47 & 4.3 & 20 \\
\hline
\end{tabular}

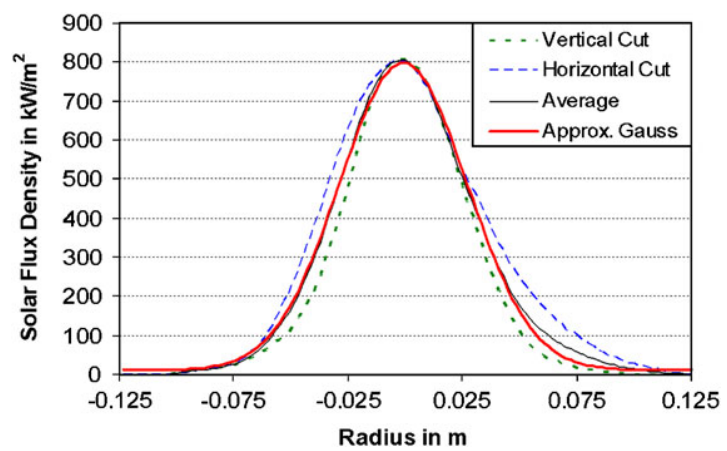

Figure 6. Distribution of the solar flux density in the solar furnace for vertical and horizontal cuts as average and approximation.

reactor, but also influence the temperature distribution in the absorber. As the chemical reaction is highly temperature sensitive, the distribution of the solar flux density has a large impact on the reactor conversion and efficiency.

The spacial distribution of solar flux provided by the solar furnace is highly inhomogeneous, see Figure 6 . The peak value is in the center of the absorber and it depends on the shutter position.

In most experiments only a part of the available solar power is needed. The shutter of the solar furnace is used to control this power by the one-dimensional, vertical movement of the two halves of a blend. Typically only $20 \%$ of the maximum opening of the shutter is applied, which means that solar radiation is pre-dominantly cut off in the top and bottom part of the beam. Therefore, the distribution is not perfectly rotationally symmetric during normal operation, see horizontal and vertical cut in Figure 6. For the model, a Gaussian distribution is used which is approximated from the average of the horizontal and vertical cut.

Approximately $90 \%$ of the solar power are absorbed in the absorber, which has a diameter of $14.4 \mathrm{~cm}$. The remaining $10 \%$ are considered as energy losses. A part of the total solar power is considered as energy loss due to the reflection and absorption at the quartz window.

The solar radiation is not only absorbed at the front surface of the absorber. Since the absorber is a porous structure, a part of the solar radiation can penetrate into the channels where it is absorbed in different depths. Each disc of the discretized absorber absorbes a certain fraction of the solar power, see Figure 7.

This correlation is derived geometrically by considering the width of a channel and the entrance angle of the radiation. 


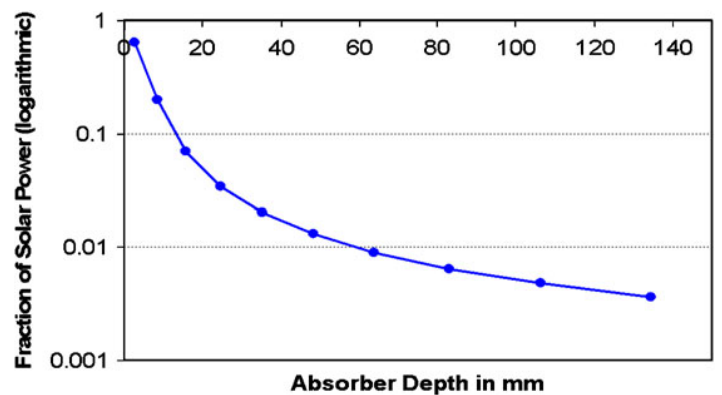

Figure 7. Fraction of the solar power absorbed inside the absorber against the absorber depth.

\subsection{Model of the re-radiation}

The TR of the absorber called re-radiation is one of the largest energy losses of the reactor. It has a large impact on the temperature distribution in the absorber and reduces the reactor efficiency.

The heat flow of TR from the front end of the absorber can be calculated as the sum of the heat flow, which are radiated to the conical duct at the reactor entrance (conus, see Figure 1), to the window and to the ambience:

$$
\dot{Q}_{\mathrm{TR}}=\dot{Q}_{\mathrm{TR}, \text { conus }}+\dot{Q}_{\mathrm{TR}, \text { window }}+\dot{Q}_{\mathrm{TR}, \text { ambience }}
$$

The three heat flows have to be calculated separately since the radiated energy depends on the temperature of the body facing the absorber.

The heat flow that is radiated to the conus is calculated with the following equation:

$$
\begin{aligned}
\dot{Q}_{\mathrm{TR}, \text { conus }}= & \phi_{13} \cdot \varepsilon_{\text {absorber }} \cdot \varepsilon_{\text {conus }} \cdot C_{S} \cdot A_{\text {absorber }} \\
& \cdot\left(T_{\text {absorber }}^{4}-T_{\text {conus }}^{4}\right)
\end{aligned}
$$

where $\phi_{13}$ is a geometrical factor that depends on the size, distance, and angle of the two interchanging surfaces of the absorber and the conus, $\varepsilon_{\text {absorber }}=0.90$ and $\varepsilon_{\text {conus }}=0.75$ are the emissivities of the absorber and the conus, $C_{S}$ is the Stefan-Boltzmann constant, $A_{\text {absorber }}$ is the surface area of the absorber, $T_{\text {absorber }}$ and $T_{\text {conus }}$ are the surface temperatures.

The equations for the heat flow to the window and to the ambience are similar. An additional factor $a_{\lambda<\lambda_{\text {Grenz }}}$ has to be included, which defines the fraction of re-radiation absorbed by the window (longer wavelengths). The remaining short-wavelength part of the TR penetrates through the window and therefore interchanges with the 'cold' environment. $\phi_{12}$ is a geometrical factor that depends on the size, distance, and angle of the two interchanging surfaces of the absorber and the ambient.

The quartz window has a certain threshold wavelength $\lambda_{\text {Grenz }}$. TR with a wavelength above this threshold wavelength is absorbed by the window. The quartz window is transparent to radiation with a shorter wavelength. The following equation describes the radiation exchange between the absorber and the ambient for this short wavelength range:

$$
\begin{aligned}
\dot{Q}_{\mathrm{TR}, \text { ambien } t}= & a_{\lambda<\lambda_{\mathrm{Grenz}}} \cdot \phi_{12} \cdot \varepsilon_{\mathrm{Absorber}} \cdot \varepsilon_{\mathrm{ambient}} \\
& \cdot C_{S} \cdot A_{\text {Absorber }} \cdot\left(T_{\text {Absorber }}^{4}-T_{\text {ambient }}^{4}\right)
\end{aligned}
$$

\subsection{Other sub-models}

Besides the above-mentioned partial models, the overall model and the partial models itself consist of a couple of other partial models. A model of the reactor housing represents the heat conduction from the absorber to the outer walls. Energy losses due to the natural convection at the housing are calculated.

The mass flow entering the reactor has to be distributed to the rings in the discretized absorber. The mass flows in the individual rings differ since the crosssection area of each ring has a different size. A homogeneous flow through each absorber channel is assumed. Owing to the different temperature in each absorber ring, the $\mathrm{SO}_{3}$-conversion at the outlet of each ring is different. The different mass flows are mixed in an ideal mixer without any energy losses. The $\mathrm{SO}_{3}$ conversion in the reactor is determined using the outlet stream of the ideal mixer.

The model was iteratively developed by stepwise addition and refinement of individual submodels. This is, in particular, true for the sub-models concerning solar radiation, radiative heat transfer and thermal reradiation.

\section{VALIDATION}

The reactor model has been set up for the test reactor, which has been investigated experimentally in the solar furnace. A validation of the model is necessary to ensure a sufficient accuracy of the simulation results.

A validation is performed by a comparison of the simulation results with the measured data in the solar furnace. The developed model is a dynamic model and allows calculating not only steady-state, but also the transient behavior of the reactor. In this first phase of validation, only states of the reactor in thermal equlibrium have been used. Each simulation is started with a reactor at an ambient temperature and constant input values and is run until all parameters are constant. The simulation results of the last time step are taken for the evaluation.

Accordingly, only such experimental results are taken for the validation for which the reactor was nearly in steady-state conditions. For process optimization purposes, the most important parameters are the conversion and the efficiency. Both parameters depend on other simulation results such as the temperature distribution in the absorber or the operating temperature. 
The correct calculation of temperature and temperature distributions needs to be proven before 'derived' values such as the average conversion and efficiency can be validated. 'Derived value' means that it depends on other parameters calculated in a simulation run, e.g. the temperture distribution of the monolith crucially influences average conversion and efficiency.

\subsection{Validation of the temperature distribu- tion}

The calculated temperature distribution in the absorber is compared with the measured temperatures. Several thermocouples were installed in the absorber during the experiments in different positions. The thermocouples were distributed in the depth of the absrober as well as in its diameter.

In Figures 8 and 9, the measured temperatures are depicted as diamonds and squares. The error bars indicate the measuring accuracy.

The temperature distribution is validated in the axial and radial direction for three different operating temperatures $\left(1000,1100\right.$ and $\left.1200^{\circ} \mathrm{C}\right)$. The operating temperature is defined as the maximum temperature in the absorber. For the axial temperature profile, the values along the center axis of the absorber are considered. For the radial profile, the temperature distributions across the irradiated surface are compared. In the experiment, the relevant thermocouples were installed in a depth of $5 \mathrm{~mm}$. This corresponds to the temperatures of the first disc of the absorber in the model.

Those six distributions (along the center axis and across the front surface for three different temperatures) were exemplarily validated and assumed to be representative for the behavior of the remaining parts of the absorber and for other operating temperatures. For example, the comparison of the axial profiles for an operating temperature of $1100^{\circ} \mathrm{C}$ is shown in Figure 8.

At the front and rear end of the absorber, the measured and calculated temperatures agree perfectly. In the middle, the simulation results are slightly lower

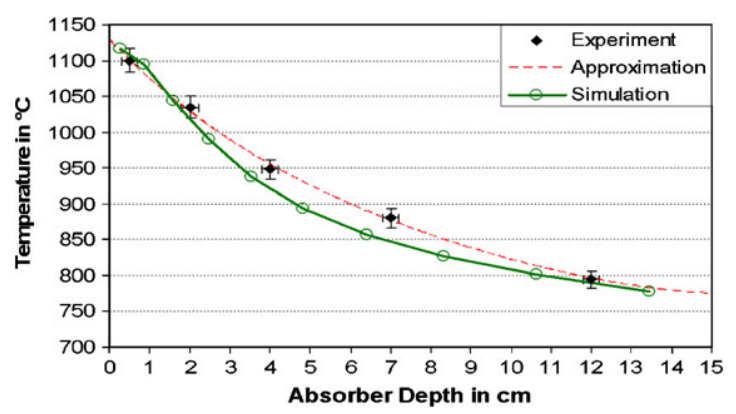

Figure 8. Comparison of the measured and simulated temperature profile in the center axis for an operating temperature of ca. $1100^{\circ} \mathrm{C}\left(P=1 \mathrm{bar}, \operatorname{Vol}\right.$. flow $\left.\left(\mathrm{N}_{2}\right)=2.2 \mathrm{Nm}^{3} \mathrm{~h}^{-1}\right)$. than the measured values. But the relative deviation is smaller than $4 \%$.

For the other two operating temperatures (1000 and $1200^{\circ} \mathrm{C}$ ), the profile is similar. The maximum relative deviation is $7 \%$. This accuracy of the model is sufficient since such deviations occur only in a small part of the absorber. A potential reason for the deviation in the middle of the absorber is a possible influence of TR in the axial direction inside the absorber, which is not considered in the model.

The radial temperature distribution in the experiments is not rotationally symmetric, since the distribution of the solar flux density in the solar furnace was slightly elliptical. Therefore, the temperature profiles in the $x$-axis and in the $y$-axis are different. The comparison of the radial temperature profiles is shown exemplarily for an operating temperature of $1200^{\circ} \mathrm{C}$ in Figure 9. The model is axially symmetric and cannot reproduce such a temperature distribution. Consequently, the calculated temperatures are in between the measured temperatures of both axes.

\subsection{Validation of the operating tempera- ture}

The operating temperature is defined as the maximum temperature in the absorber. In the experiments, it corresponds to the temperature that is measured with the thermocouple in the center axis in a depth of $5 \mathrm{~mm}$ from the irradiated surface. In the simulation, this is consequently the temperature of the center volume element of the first disc. The operating temperature is mainly dependent on the solar power input and on the total volume flow through the reactor. Both correlations are validated.

The simulated and measured operating temperatures are shown against the irradiated solar power in Figure 10.

The total volume flow shown in the experiments was similar, but not exactly the same due to operational reasons. The simulation results are in most cases within the limits of measuring accuracy of the thermocouple. This indicates a sufficient high representation accuracy of the model.

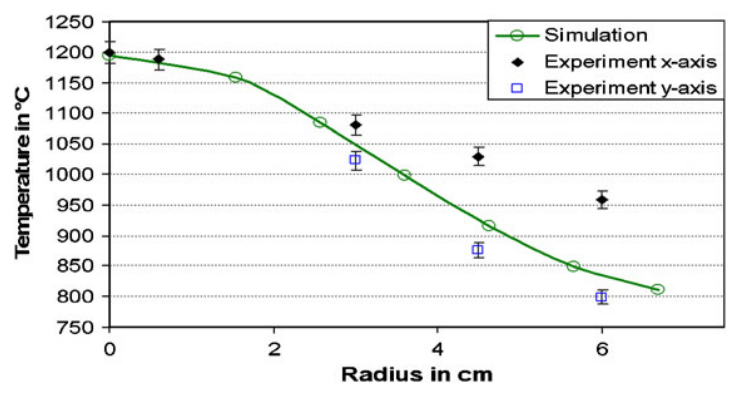

Figure 9. Comparison of the measured and simulated temperature profile on the irradiated surface of the absorber in the radial direction for an operating temperature of ca. $1200^{\circ} \mathrm{C}$, $\left(P=1\right.$ bar, Vol. flow $\left.\left(\mathrm{N}_{2}\right)=2.2 \mathrm{Nm}^{3} \mathrm{~h}^{-1}\right)$. 


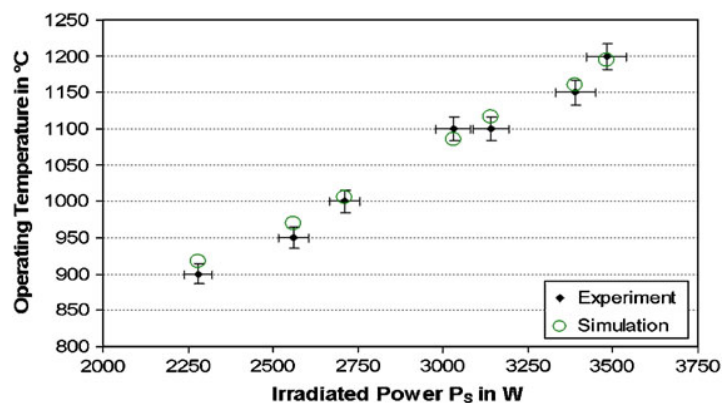

Figure 10. Comparison of the measured and simulated operating temperature against the irradiated power PS for different volume flows $\left(P=1\right.$ bar, $\operatorname{Vol}$. flow $\left.\left(\mathrm{N}_{2}\right)=2.2 \mathrm{Nm}^{3} \mathrm{~h}^{-1}\right)$.

The correlation between the operating temperature and the total volume flow is not shown here but is represented with similar accuracy by the model.

\subsection{Validation of the conversion}

The $\mathrm{SO}_{3}$-conversion in the reactor is mainly influenced by the solar power input and the total volume flow. The latter determines (together with the temperature distribution) the residence time in the presence of the catalyst. The correlation between the solar power and the operating temperature is evaluated and proven above. Thus, the accuracy of calculating the conversion can be validated by inspecting its dependency on the operating temperature instead of the solar power.

In Figure 11 the experimentally determined conversion is plotted and compared with the conversions calculated by means of the reactor model. The simulated values are inside the determination accuracy of the experimental data.

Deviations exist with respect to both axes. Regarding the $x$-axis, the calculated operating temperature deviates from the measured one. At the $y$-axis, deviations of the calculated conversions from the experiments can be read off.

The comparison of the correlation between the conversion and the total volume flow shows also a sufficient accuracy of the model.

\subsection{Validation of the efficiency}

The validation of an adequate reproduction of the reactor efficiency is highly important since it proves that the sub-models of all physical and chemical processes in the overall model are suitable. It also proves that no important processes have been omitted, since the efficiency depends on more or less all processes in the reactor and on all relevant process parameters. The reactor efficiency $\eta_{\text {reactor }}$ is defined as the ratio of the net heat flow $\dot{Q}_{\text {net,reactor }}$ to the solar irradiated power $P_{\text {solar }}$. The net heat flow is defined as the sum of the heat flow, which is required for the dissociation of $\mathrm{H}_{2} \mathrm{SO}_{4}$, the decomposition of $\mathrm{SO}_{3}$ and

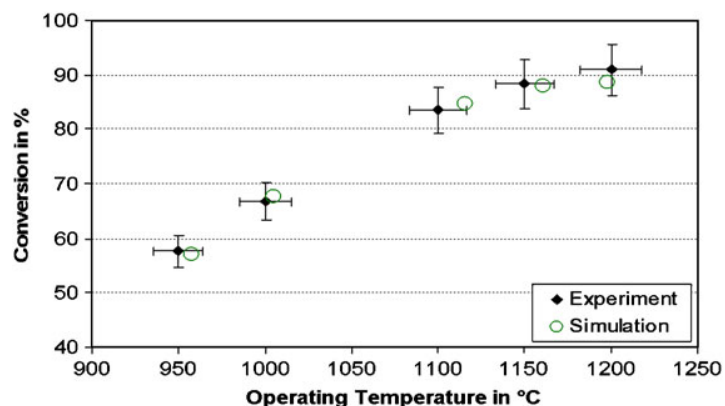

Figure 11. Comparison of the measured and simulated conversions vs the operating temperature $(P=1 \mathrm{bar}$, Vol flow $\left(\mathrm{N}_{2}\right)=2.2 \mathrm{Nm}^{3} \mathrm{~h}^{-1}$, Vol. flow $\left.\left(\mathrm{H}_{2} \mathrm{SO}_{4}, \mathrm{liq}\right)=3-5 \mathrm{ml} \mathrm{min}^{-1}\right)$.

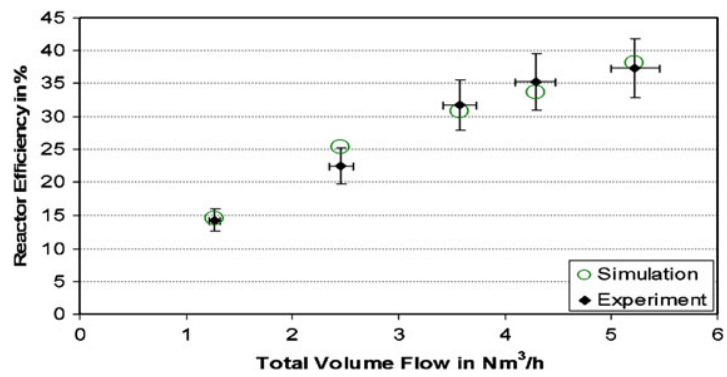

Figure 12. Comparison of the simulated and experimentally gained reactor efficiencies vs total volume flow through the reactor $\left(T_{\text {operating }}=900^{\circ} \mathrm{C}-1200^{\circ} \mathrm{C}, P=1\right.$ bar, $\operatorname{Vol}$. flow $\left(\mathrm{N}_{2}\right)=$ $0.5-2.2 \mathrm{Nm}^{3} \mathrm{~h}^{-1}$, Vol. flow $\left.\left(\mathrm{H}_{2} \mathrm{SO}_{4}, \mathrm{liq}\right)=3-5 \mathrm{ml} \mathrm{min}{ }^{-1}\right)$.

the heating up of the gases:

$$
\begin{aligned}
\eta_{\text {reactor }} & =\frac{\dot{Q}_{\text {net,reactor }}}{P_{\text {solar }}} \\
& =\frac{\dot{Q}_{\mathrm{H}_{2} \mathrm{SO}_{4}-\text { dissociation }}+\dot{Q}_{\mathrm{SO}_{3} \text {-decomposition }}+\dot{Q}_{\text {sensibleheat }}}{P_{\text {solar }}}
\end{aligned}
$$

Exemplarily, the dependancy of the reactor efficiency on the total volume flow is shown in Figure 12. The experiments and the simulation show an increasing efficiency with increasing volume flow. The deviations between the experiment and simulation are sufficiently small to have an adequate representation. Figure 9 indicates that in the experiments, which were carried out with maximum volume flows of about $5.5 \mathrm{Nm}^{3} \mathrm{~h}^{-1}$, the possible optimum of reactor efficieny obiously was not yet reached. The model is now systematically applied to identify such and comparable ways to improve and optimize process and hardware.

\section{CONCLUSIONS}

A dynamic overall model of a volumetric receiver-reactor for the decomposition of sulfuric acid has been 
developed. The model contains calculations of the most important processes such as the absorption of the solar radiation, heat transfer from the absorber to the process gas, TR at the absorber surfaces and inside the absorber channels and particularly the reaction kinetics of the $\mathrm{SO}_{3}$ decomposition. An appropriate discretization of the absorber allows a fast simulation without numerical problems.

A validation of the model has been performed successfully. The most important correlations between input and output parameters were checked. The relative deviations between the experimental data and the simulation results are within the range of measurement accuracy. In particular, the good agreement of calculated values of the derived parameters, $\mathrm{SO}_{3}$ conversion and reactor efficiency, with those determined from the experiments qualifies the model for optimization purposes.

Meanwhile a comprehensive optimization of process parameters and the reactor design has been carried out using the described model and will be published in a separate paper.

\section{ACKNOWLEDGEMENTS}

The authors thank the European Commission for cofunding the project HYTHEC in the sixth framework program (contract number 502704).

\section{REFERENCES}

1. Turner J, Sverdrup G, Mann MK, Maness PC, Kroposki B, Ghirardi M, Evans RJ, Blake D. Renewable hydrogen production. International Journal of Energy Research 2008; 32(5):379-407. DOI: $10.1002 /$ er. 1372 .

2. Norman J, Besenbruch GE, Brown LC, O'Keefe DR, Allen CL. Thermochemical water-splitting cycle. Bench-scale investigations and process engineering. DOE Report DOE/ET/26225-1, 1982.

3. Brecher LE, Spewock S, Warde CJ. The Westinghouse sulfur cycle for the thermochemical decomposition of water. In Proceedings of the 1st World Hydrogen Energy Conference, Miami Beach, FL, U.S.A., Veziroglu TN (ed.), vol. 1, 1976; 9A-1-9A-16.

4. Monnerie N, Müller-Steinhagen H, Roeb M, Sattler C, Schmitz M. Hydrogen production by solar thermochemical water splitting. Seventh World Congress of Chemical Engineering, Glasgow, U.K., 10-14 July 2005.

5. Neumann A, Groer U. Experimenting with concentrated sunlight using the DLR solar furnace. Solar Energy 1996; 58:181-190.

6. Noglik A, Roeb M, Sattler C, Pitz-Paal R. Experimental study on sulfur trioxide decomposition in a volumetric solar receiver-reactor. International
Journal of Energy Research 2009; 33(9):799-812. DOI: $10.1002 /$ er. 1514 .

7. Roeb M, Noglik A, Monnerie N, Schmitz M, Sattler C, Cerri G, de Maria G, Giovannelli A, Orden A, de Lorenzo D, Cedillo J, LeDuigou A, Borgard JM. Development and verification of process concepts for the splitting of sulfuric acid by concentrated solar radiation. Proceedings of the 13th SolarPACES International Symposium, Seville, Spain, 20-23 June 2006.

8. Le Duigou A, Borgard JM, Larousse B, Doizi D, Allen RWK, Ewan BC, Priestman GH, Elder R, Devonshire R, Ramos V, Cerri G, Salvini C, Giovannelli A, De Maria G, Corgnale C, Brutti S, Roeb M, Noglik A, Rietbrock P, Mohr S, de Oliveira L, Monnerie N, Schmitz M, Sattler C, Orden Martinez A, de Lorenzo Manzano D, Cedillo Rojas J, Dechelotte S, Baudouin O. HYTHEC: an EC funded search for a long term massive hydrogen production route using solar and nuclear technologies. International Journal of Hydrogen Energy 2007; 32:1516-1529. DOI: 10.1016/j.ijhydene.2006.10.047.

9. Noglik A, Roeb M, Rzepczyk T, Sattler C, Pitz-Paal R. solar thermochemical generation of hydrogen: development of a receiver reactor for the decomposition of sulfuric acid. ASME International Conference on Energy Sustainabililty (ES2007), Long Beach, CA, U.S.A., 27-30 June 2007.

10. Noglik A, Roeb M, Rzepczyk T, Hinkley J, Sattler C, Pitz-Paal R. Solar thermochemical generation of hydrogen: development of a receiver reactor for the decomposition of sulfuric acid. Journal of Solar Energy Engineering 2009; 131:011003.1-011003.7. DOI:10.1115/1.3027505.

11. Noglik A, Roeb M, Sattler C, Pitz-Paal R. Experimental study on sulfur trioxide decomposition in a volumetric solar receiver-reactor. ASME 2nd International Conference on Energy Sustainabililty (ES2008), Jacksonville, Fl, U.S.A., 10-14 August 2008.

12. Noglik A. Entwicklung eines solaren Reaktors zur Schwefelsäurespaltung für die thermochemische Wasserstofferzeugung. Fortschrittsberichte VDI-Reihe $3 \mathrm{Nr}$. 896. VDI-Verlag: Düsseldorf, Germany, 2008. ISBN 978-3-18-389603-5, ISSN 0178-9503.

13. Knoche KF. Thermochemical H2-production with a solar driven sulfur-iodine-process. In Solar Thermal Energy Utilization, M Becker, K-H Funken (eds), vol. 4. Springer: Berlin, 1988; 441-498.

14. Lüpfert E. Der Einfluss konzentrierter Solarstrahlung auf die Reaktionsgeschwindigkeit der Abfallschwefelsäure-Spaltung. Dissertation RWTH Aachen, 1996.

15. General Atomics. Decomposition of sulfuric acid using solar thermal energy. General Atomics Report, $G A-A-17573,1985$. 
16. Bilgen ER, Joels RK. An assessment of solar hydrogen production using the Mark 13 Hybrid process. International Journal of Hydrogen Energy 1985; 10:143-155.

17. Poitou S, Rodriguez G, Haquet N, Cachon L, Bucci P, Tochon P, Chaumat V, Gillia O, Gwinner B, Balbaud F, de Lamare J. Development program of the SO3 decomposer as a key component of the sulphur based thermochemical cycles: new steps towards feasibility demonstration. Proceeding of the 17th World Hydrogen Energy Conference (WHEC), Brisbane, Australia, 19-23 June 2008.

18. Kolb G, Diver RB, Siegel N. Central-station solar hydrogen power plant. Journal of Solar Energy Engineering 2007; 129:179-183. DOI: 10.1115/1.2710246.
19. Dersch J, Mathijssen A, Roeb M, Sattler C. Modelling of a solar thermal reactor for hydrogen generation. Fifth Modelica Conference, Vienna, Austria, 2006.

20. Roeb M, Neises M, Säck JP, Rietbrock P, Monnerie N, Dersch J, Schmitz M, Sattler C. Operational strategy of a two-step thermochemical process for solar hydrogen production. International Journal of Hydrogen Energy 2009; 34(10):4537-4545. DOI: 10.1016/j.ijhydene.2008. 08.049 .

21. Modellica - a unified object-oriented language for physical system. Modelling Language Specification Version 2.2, Modelica Association, 2005.

22. Grell J. Reaktionskinetische Untersuchung zur katalytischen Reduktion von Schwefeltrioxid. Bachelor Thesis, Fachhochschule Bonn-Rhein-Sieg, 2006. 